\title{
ERRATUM TO \\ VON NEUMANN SPECTRA NEAR ZERO
}

\author{
M. Gromov, M. Shubin
}

Proposition 3.1 in [GS] is false. (We missed in the proof that the forms with compact support are not sufficient to evaluate $F_{k}(\lambda ; M)$.) However, Corollary 3.1 where Proposition 3.1 was used remains valid. The correct argument, using the elliptic boundary value problems, was suggested to us earlier by V. Ivrii (and our "proof" was due to an attempt to circumvent Ivrii's argument). Also a correct proof of the finiteness of $F_{k}(\lambda ; M)$, similar to that by Ivrii and using the double trick, appears in [LLü, Sect. 5].

On the other hand none of our arguments use the finiteness of $F_{k}(\lambda ; M)$ and none of the proofs in our paper, except that of Corollary 3.1, need any change.

We are grateful to J. Lott and A. Mishchenko who indicated the error.

\section{References}

[GS] M. Gromov, M. ShubIN, Von Neumann spectra near zero, Geometric And Functional Analysis (GAFA) 1:4 (1991), 375-404.

[LLï] J. LotT, W.LücK, $L^{2}$-topological invariants of 3-manifolds, Inventiones Math. 120:1 (1995), 15-60.

M. Gromov

IHES

35 route de Chartres 91440 Bures sur Yvette France
M. Shubin

Dept. of Math.

Northeastern University

Boston, MA 02115

USA 